\title{
More precise Pair Correlation Conjecture on the zeros of the Riemann zeta function
}

\author{
by
}

Tsz Ho Chan (Cleveland, OH)

1. Introduction. In the early 1970s, H. L. Montgomery studied the distribution of the differences $\gamma-\gamma^{\prime}$ between the imaginary parts of the non-trivial zeros of the Riemann zeta function. Let

$$
F(x, T)=\sum_{\substack{0 \leq \gamma \leq T \\ 0 \leq \gamma^{\prime} \leq T}} x^{i\left(\gamma-\gamma^{\prime}\right)} w\left(\gamma-\gamma^{\prime}\right), \quad w(u)=\frac{4}{4+u^{2}} .
$$

Assuming the Riemann Hypothesis, he proved in [8] that, as $T \rightarrow \infty$,

$$
F(x, T) \sim \frac{T}{2 \pi} \log x+\frac{T}{2 \pi x^{2}}(\log T)^{2}
$$

for $1 \leq x \leq T$ (actually he only proved this for $1 \leq x \leq o(T)$ and the full range was done by Goldston [4]). He conjectured that

$$
F(x, T) \sim \frac{T}{2 \pi} \log T
$$

for $T \leq x \leq T^{M}$, $M$ fixed, which is known as the Strong Pair Correlation Conjecture. From this, one has the (Weak) Pair Correlation Conjecture:

$$
\sum_{\substack{0<\gamma, \gamma^{\prime} \leq T \\ 0<\gamma-\gamma^{\prime} \leq 2 \pi \alpha / \log T}} 1 \sim \frac{T}{2 \pi} \log T \int_{0}^{\alpha}\left[1-\left(\frac{\sin \pi u}{\pi u}\right)^{2}\right] d u .
$$

In [1], the author proved that, under the Riemann Hypothesis, for any $\varepsilon>0$,

$$
\begin{aligned}
F(x, T)= & \frac{1}{2 \pi} T \log x+\frac{1}{x^{2}}\left[\frac{T}{2 \pi}\left(\log \frac{T}{2 \pi}\right)^{2}-2 \frac{T}{2 \pi} \log \frac{T}{2 \pi}\right] \\
& +O(x \log x)+O\left(\frac{T}{x^{1 / 2-\varepsilon}}\right)
\end{aligned}
$$

2000 Mathematics Subject Classification: Primary 11M26. 
for $1 \leq x \leq T / \log T$. This gives a more precise formula for $F(x, T)$ in the range $1 \leq x \leq T / \log T$. Meanwhile, in [2], the author derived a more precise Strong Pair Correlation Conjecture: For every fixed $\varepsilon>0$ and $A \geq 1+\varepsilon$,

$$
F(x, T)=\frac{T}{2 \pi} \log \frac{T}{2 \pi}-\frac{T}{2 \pi}+O\left(T^{1-\varepsilon_{1}}\right)
$$

holds uniformly for $T^{1+\varepsilon} \leq x \leq T^{A}$ with some $\varepsilon_{1}>0$. It remains to understand how $F(x, T)$ changes from (2) to (3) when $x$ is near to $T$. For that, we prove

Theorem 1.1. Assume the Riemann Hypothesis and the Twin Prime Conjecture in the form stated at the start of Section 2. For any small $\varepsilon>0$ and any integer $M>2$,

$$
\begin{aligned}
F(x, T)= & \frac{T}{2 \pi} \log x-\frac{4 x}{3 \pi} \int_{0}^{T / x} \frac{\sin v}{v} d v+\frac{x^{2}}{\pi T}\left(\sum_{h=1}^{\infty} \frac{\mathfrak{S}(h)}{h^{2}}\right)\left(1-\cos \frac{T}{x}\right) \\
& -\frac{x}{2 \pi} \int_{1}^{\infty} \frac{\sin \frac{T y}{x}}{y^{2}} d y+\left(\frac{B}{2}+\frac{11}{12}\right) \frac{x}{\pi} \int_{1}^{\infty} \frac{\sin \frac{T y}{x}}{y^{4}} d y \\
& -\frac{4 T}{\pi} \int_{1}^{\infty} \frac{f(y)}{y^{2}} \frac{\sin \frac{T}{x} y}{\frac{T}{x} y} d y+\frac{2 T}{\pi} \int_{1}^{\infty} \frac{\int_{1}^{y} f(u) d u}{y^{3}} \frac{\sin \frac{T}{x} y}{\frac{T}{x} y} d y \\
& +\frac{6 T}{\pi} \int_{1}^{\infty} y \int_{y}^{\infty} \frac{f(u)}{u^{4}} d u \frac{\sin \frac{T}{x} y}{\frac{T}{x} y} d y+O\left(\frac{x^{1+6 \varepsilon}}{T}\right) \\
& +O\left(x^{1 / 2+7 \varepsilon}\right)+O\left(\frac{T}{(\log T)^{M-2}}\right),
\end{aligned}
$$

for $T /(\log T)^{M} \leq x \leq T^{2-\varepsilon}$. Here $B=-C_{0}-\log 2 \pi, C_{0}$ is Euler's constant $0.5772156649 \ldots$, and $\mathfrak{S}(h)$ and $f(u)$ are defined in the next section. The implicit constants may depend on $\varepsilon$ and $M$.

As corollaries of Theorem 1.1, we have:

Corollary 1.1. Assume the Riemann Hypothesis and the Twin Prime Conjecture. For any integer $M>2$,

for $T /(\log T)^{M} \leq x \leq T$.

$$
F(x, T)=\frac{T}{2 \pi} \log x+O(x)+O_{M}\left(\frac{T}{(\log T)^{M-2}}\right)
$$

Corollary 1.2. Assume the Riemann Hypothesis and the Twin Prime Conjecture. For any small $\varepsilon>0$ and any integer $M>2$,

$$
F(x, T)=\frac{T}{2 \pi} \log \frac{T}{2 \pi}-\frac{T}{2 \pi}+O_{\varepsilon}\left(T\left(\frac{T}{x}\right)^{1 / 2-\varepsilon}\right)+O_{\varepsilon, M}\left(\frac{T}{(\log T)^{M-2}}\right)
$$

for $T \leq x \leq T^{2-29 \varepsilon}$. 
This work is part of the author's 2002 Ph.D. thesis. The author would also like to thank the referee for some helpful suggestions.

2. Preparations. We mentioned the Twin Prime Conjecture in the previous section. The form needed is the following: For any $\varepsilon>0$,

$$
\sum_{n=1}^{N} \Lambda(n) \Lambda(n+d)=\mathfrak{S}(d) N+O\left(N^{1 / 2+\varepsilon}\right) \quad \text { uniformly in }|d| \leq N .
$$

Here $\Lambda(n)$ is the von Mangoldt lambda function and

$$
\mathfrak{S}(d)=2 \prod_{p>2}\left(1-\frac{1}{(p-1)^{2}}\right) \prod_{p \mid d, p>2} \frac{p-1}{p-2} \quad \text { if } d \text { is even, }
$$

and $\mathfrak{S}(d)=0$ if $d$ is odd. We also need a lemma concerning $\mathfrak{S}(d)$.

Lemma 2.1. For any $\varepsilon>0$,

$$
\sum_{k=1}^{h}(h-k) \mathfrak{S}(k)=\frac{1}{2} h^{2}-\frac{1}{2} h \log h+A h+O\left(h^{1 / 2+\varepsilon}\right),
$$

where $A=\frac{1}{2}\left(1-C_{0}-\log 2 \pi\right)$ and $C_{0}$ is Euler's constant.

Proof. This is a theorem in Montgomery and Soundararajan [9] and is also found in Friedlander and Goldston [3].

Borrowing from [6], we define

Then from [3],

$$
\begin{aligned}
& S_{\alpha}(y):=\sum_{h \leq y} \mathfrak{S}(h) h^{\alpha}-\frac{y^{\alpha+1}}{\alpha+1} \quad \text { for } \alpha \geq 0, \\
& T_{\alpha}(y):=\sum_{h>y} \frac{\mathfrak{S}(h)}{h^{\alpha}} \quad \text { for } \alpha>1 .
\end{aligned}
$$

$$
S_{0}(y)=-\frac{1}{2} \log y+O\left((\log y)^{2 / 3}\right) .
$$

Suppose $S_{0}(y)=-\frac{1}{2} \log y+\varepsilon(y)$. By partial summation,

$$
\begin{aligned}
& S_{\alpha}(y)=-\frac{y^{\alpha}}{2 \alpha}+\varepsilon(y) y^{\alpha}-\alpha \int_{1}^{y} \varepsilon(u) u^{\alpha-1} d u+\left(\frac{1}{2 \alpha}+\frac{\alpha}{\alpha+1}\right), \\
& T_{\alpha}(y)=\frac{1}{(\alpha-1) y^{\alpha-1}}-\frac{\varepsilon(y)}{y^{\alpha}}-\frac{1}{2 \alpha y^{\alpha}}+\alpha \int_{y}^{\infty} \frac{\varepsilon(u)}{u^{\alpha+1}} d u .
\end{aligned}
$$

Lemma 2.2. For any $\varepsilon>0$,

$$
\int_{1}^{y} \varepsilon(u) d u=\frac{B}{2} y+O\left(y^{1 / 2+\varepsilon}\right)
$$

where $B=-C_{0}-\log 2 \pi$ as in the previous section. 
Proof. By Lemma 2.1,

$$
\begin{aligned}
\int_{1}^{y} \varepsilon(u) d u & =\int_{1}^{y}\left(\sum_{h \leq u} \mathfrak{S}(h)-u+\frac{1}{2} \log u\right) d u \\
& =\sum_{h \leq y}(y-h) \mathfrak{S}(h)-\frac{1}{2} y^{2}+\frac{1}{2} y \log y-\frac{1}{2} y+1 \\
& =A y-\frac{1}{2} y+O\left(y^{1 / 2+\varepsilon}\right)=\frac{B}{2} y+O\left(y^{1 / 2+\varepsilon}\right) .
\end{aligned}
$$

Now, let us define

$$
f(y):=\int_{1}^{y}\left(\varepsilon(u)-\frac{B}{2}\right) d u
$$

By integration by parts and Lemma 2.2, one has

$$
\begin{aligned}
& \int_{1}^{y} \varepsilon(u) u d u=\frac{B}{4} y^{2}+y f(y)-\int_{1}^{y} f(u) d u-\frac{B}{4}=\frac{B}{4} y^{2}+O\left(y^{3 / 2+\varepsilon}\right), \\
& \int_{y}^{\infty} \frac{\varepsilon(u)}{u^{3}} d u=\frac{B}{4 y^{2}}-\frac{f(y)}{y^{3}}+3 \int_{y}^{\infty} \frac{f(u)}{u^{4}} d u=\frac{B}{4 y^{2}}+O\left(y^{-5 / 2+\varepsilon}\right) .
\end{aligned}
$$

We also need the following lemmas.

Lemma 2.3. Assume the Riemann Hypothesis. For any $\varepsilon>0$,

$$
\begin{aligned}
& \sum_{n \leq x} \Lambda(n)^{2} n=\frac{1}{2} x^{2} \log x-\frac{1}{4} x^{2}+O\left(x^{3 / 2+\varepsilon}\right), \\
& \sum_{n>x} \frac{\Lambda(n)^{2}}{n^{3}}=\frac{1}{2} \frac{\log x}{x^{2}}+\frac{1}{4} \frac{1}{x^{2}}+O\left(\frac{1}{x^{5 / 2-\varepsilon}}\right),
\end{aligned}
$$

where the implicit constants may depend on $\varepsilon$.

Proof. This follows by partial summation and prime number theorem under the Riemann Hypothesis.

Lemma 2.4. For any $x \geq 1$, and $f(u)$ defined in (7), we have

$$
\sum_{h \geq x} \frac{\mathfrak{S}(h)}{h^{2}} \ll \frac{1}{x}, \quad \sum_{h=1}^{\infty} \frac{\mathfrak{S}(h)}{h^{2}}=\frac{7}{4}+\frac{B}{2}+6 \int_{1}^{\infty} \frac{f(u)}{u^{4}} d u,
$$

where $B=-C_{0}-\log 2 \pi$ and $C_{0}$ is Euler's constant again.

Proof. First, from (4),

$$
\sum_{h \geq x} \frac{\mathfrak{S}(h)}{h^{2}}=\int_{x}^{\infty} \frac{1}{u^{2}} d\left(S_{0}(u)+u\right) \ll \frac{1}{x}+\int_{x}^{\infty} \frac{\log u}{u^{3}} d u \ll \frac{1}{x} .
$$


So, the series converges and equals

$$
\begin{aligned}
\int_{1}^{\infty} \frac{1}{u^{2}} d\left(S_{0}(u)+u\right) & =2 \int_{1}^{\infty} \frac{S_{0}(u)+u}{u^{3}} d u=2 \int_{1}^{\infty} \frac{u-\frac{1}{2} \log u+\varepsilon(u)}{u^{3}} d u \\
& =2-\frac{1}{4}+2 \int_{1}^{\infty} \frac{\varepsilon(u)}{u^{3}} d u=\frac{7}{4}+\frac{B}{2}+2 \int_{1}^{\infty} \frac{\varepsilon(u)-\frac{B}{2}}{u^{3}} d u \\
& =\frac{7}{4}+\frac{B}{2}+2 \int_{1}^{\infty} \frac{1}{u^{3}} d f(u)=\frac{7}{4}+\frac{B}{2}+6 \int_{1}^{\infty} \frac{f(u)}{u^{4}} d u
\end{aligned}
$$

by integration by parts and the definitions of $\varepsilon(u)$ and $f(u)$.

3. Smooth weight. Fix a small positive real number $\varepsilon$ and let $K$ be a large integer depending on $\varepsilon$. Let $M$ be an integer greater than 2 and $U=(\log T)^{M}$. We want to define a smooth weight function $\Psi_{U}(t)$ with:

1. support in $[-1 / U, 1+1 / U]$,

2. $0 \leq \Psi_{U}(t) \leq 1$,

3. $\Psi_{U}(t)=1$ for $1 / U \leq t \leq 1-1 / U$,

4. $\Psi_{U}^{(j)}(t) \ll U^{j}$ for $j=1, \ldots, K$.

Let $\Delta=1 / 2^{K} U$. We define a sequence of functions as follows (Vinogradov's construction):

$$
\begin{aligned}
& \chi_{0}(t)= \begin{cases}1 & \text { if } 0 \leq t \leq 1 \\
0 & \text { else },\end{cases} \\
& \chi_{i}(t)=\frac{1}{2 \Delta} \int_{-\Delta}^{\Delta} \chi_{i-1}(t+x) d x \quad \text { for } i=1, \ldots, K+1 .
\end{aligned}
$$

Clearly, $0 \leq \chi_{i}(t) \leq 1$ for $1 \leq i \leq K+1$. One can easily check by induction that $\chi_{i}(t)=1$ for $2^{i-1} \Delta \leq t \leq 1-2^{i-1} \Delta$, and $\chi_{i}(t)=0$ for $t<-2^{i-1} \Delta$ or $t>1+2^{i-1} \Delta$ for $i=1, \ldots, K+1$.

LEMma 3.1. $\chi_{i}^{(j)}(t)$ exist and are continuous, and $\chi_{i}^{(j)}(t) \leq \Delta^{-j}$ for $0 \leq j<i \leq K+1$.

Proof. Induction on $i$. First note that $\chi_{1}(t)$ is continuous because

$$
\begin{aligned}
\left|\chi_{1}(t+\delta)-\chi_{1}(t)\right| & =\left|\frac{1}{2 \Delta} \int_{-\Delta}^{\Delta} \chi_{0}(t+\delta+x) d x-\frac{1}{2 \Delta} \int_{-\Delta}^{\Delta} \chi_{0}(t+x) d x\right| \\
& =\left|\frac{1}{2 \Delta} \int_{-\Delta+\delta}^{\Delta+\delta} \chi_{0}(t+x) d x-\frac{1}{2 \Delta} \int_{-\Delta}^{\Delta} \chi_{0}(t+x) d x\right|
\end{aligned}
$$




$$
=\left|\frac{1}{2 \Delta} \int_{\Delta}^{\Delta+\delta} \chi_{0}(t+x) d x-\frac{1}{2 \Delta} \int_{-\Delta}^{-\Delta+\delta} \chi_{0}(t+x) d x\right| \leq \frac{\delta}{\Delta} .
$$

Secondly,

$$
\begin{aligned}
\frac{\chi_{2}(t+h)-\chi_{2}(t)}{h} & =\frac{1}{h}\left[\frac{1}{2 \Delta} \int_{\Delta}^{\Delta+h} \chi_{1}(t+x) d x-\frac{1}{2 \Delta} \int_{-\Delta}^{-\Delta+h} \chi_{1}(t+x) d x\right] \\
& =\frac{1}{2 \Delta}\left[\chi_{1}\left(t+\Delta+\xi_{1}\right)-\chi_{1}\left(t-\Delta+\xi_{2}\right)\right]
\end{aligned}
$$

for some $0 \leq \xi_{1}, \xi_{2} \leq h$ by the mean-value theorem. So $\chi_{2}^{\prime}(t)$ exists and equals $\frac{1}{2 \Delta}\left[\chi_{1}(t+\Delta)-\chi_{1}(t-\Delta)\right]$, which is continuous and $\leq 1 / \Delta$. Assume that $\chi_{i}^{(j)}(t)$ are continuous and satisfy $\chi_{i}^{(j)} \ll \Delta^{-j}$ for some $2 \leq i \leq K$ and all $0 \leq j \leq i-1$. Now, for $0 \leq j \leq i-1, \chi_{i+1}^{(j)}(t)=\frac{1}{2 \Delta} \int_{-\Delta}^{\Delta} \chi_{i}^{(j)}(t+x) d x \leq \Delta^{-j}$ by the induction hypothesis. For $j=i$,

$$
\begin{aligned}
\chi_{i+1}^{(i)}(t) & =\lim _{h \rightarrow 0} \frac{\chi_{i+1}^{(i-1)}(t+h)-\chi_{i+1}^{(i-1)}(t)}{h} \\
& =\lim _{h \rightarrow 0} \frac{1}{h}\left[\frac{1}{2 \Delta} \int_{\Delta}^{\Delta+h} \chi_{i}^{(i-1)}(t+x) d x-\frac{1}{2 \Delta} \int_{-\Delta}^{-\Delta+h} \chi_{i}^{(i-1)}(t+x) d x\right] \\
& =\frac{1}{2 \Delta}\left[\chi_{i}^{(i-1)}(t+\Delta)-\chi_{i}^{(i-1)}(t-\Delta)\right],
\end{aligned}
$$

which is continuous and $\leq \Delta^{-i}$ by induction hypothesis.

LEMMA 3.2. We have

$$
\widehat{\chi}_{0}(y)=e^{\pi i y} \frac{\sin \pi y}{\pi y}, \quad \widehat{\chi}_{i+1}(y)=\widehat{\chi}_{i}(y) \frac{\sin 2 \pi \Delta y}{2 \pi \Delta y}
$$

for $0 \leq i \leq K$. Here $\widehat{f}(y)$ denotes the inverse Fourier transform of $f(t)$, $\widehat{f}(y)=\int_{-\infty}^{\infty} f(t) e(y t) d t$.

Note. We use inverse Fourier transform so that the notation matches [5] and [6].

Proof. Indeed,

$$
\widehat{\chi}_{0}(y)=\int_{0}^{1} e(y t) d t=\frac{e^{2 \pi i y}-1}{2 \pi i y}=e^{\pi i y} \frac{\sin \pi y}{\pi y} .
$$

Moreover,

$$
\widehat{\chi}_{i+1}(y)=\int_{-\infty}^{\infty} \chi_{i+1}(t) e(y t) d t=\frac{1}{2 \Delta} \int_{-\Delta}^{\Delta} \int_{-\infty}^{\infty} \chi_{i}(t+x) e(y t) d t d x
$$




$$
=\frac{1}{2 \Delta} \int_{-\Delta}^{\Delta} \widehat{\chi}_{i}(y) e(-y x) d x=\frac{\widehat{\chi}_{i}(y)}{2 \Delta} \frac{e(-y \Delta)-e(y \Delta)}{-2 \pi i y}=\widehat{\chi}_{i}(y) \frac{\sin 2 \pi \Delta y}{2 \pi \Delta y} .
$$

Now we take $\Psi_{U}(t)=\chi_{K+1}(t)$. Then $\Psi_{U}(t)$ has the required properties by the above discussion and Lemma 3.1. From Lemma 3.2, we know that

$$
\widehat{\Psi}_{U}(y)=e^{\pi i y} \frac{\sin \pi y}{\pi y}\left(\frac{\sin 2 \pi \Delta y}{2 \pi \Delta y}\right)^{K+1} .
$$

It follows that

$$
\begin{aligned}
& \operatorname{Re} \widehat{\Psi}_{U}(y)=\frac{\sin 2 \pi y}{2 \pi y}\left(\frac{\sin 2 \pi \Delta y}{2 \pi \Delta y}\right)^{K+1}, \\
& \widehat{\Psi}_{U}(y) \ll y^{-K} \quad \text { for } y \gg T^{\varepsilon}, \\
& \widehat{\Psi}_{U}(T y) \ll T^{-K \varepsilon} \quad \text { for } y \gg \tau^{-1} \text { where } \tau=T^{1-\varepsilon} .
\end{aligned}
$$

These are similar to (18) and (19) in [5]. Also, by Lemma 3.1, it follows from the discussion in [5] that

$$
\widehat{\Psi}_{U}(y), \widehat{\Psi}_{U}^{\prime}(y) \ll \min \left(1,\left(\frac{U}{2 \pi y}\right)^{K}\right)
$$

which is (17) in [5]. Consequently, the results in [5] are true with our choice of $\Psi_{U}(t)$. Moreover, if one follows their arguments carefully, one has their Corollaries 1 and 2 (except an extra $N^{\varepsilon}$ to the error terms) and Theorem 3 as long as $\tau=T^{1-\varepsilon} \leq x$.

We shall need the following lemmas concerning our weight function $\Psi_{U}(t)$. Here we assume $T \Delta \leq x$.

Lemma 3.3. For any integer $n \geq 1$,

$$
\int_{1}^{\infty} \frac{1}{y^{n}} \operatorname{Re} \widehat{\Psi}_{U}\left(\frac{T y}{2 \pi x}\right) d y=\frac{x}{T} \int_{1}^{\infty} \frac{\sin \frac{T y}{x}}{y^{n+1}} d y+O\left(K \Delta \log \frac{1}{\Delta}\right) .
$$

Proof. By a change of variable $v=\frac{T y}{x}$ and (10), the left hand side is

$$
\begin{aligned}
& \left(\frac{T}{x}\right)^{n-1} \int_{T / x}^{\infty} \frac{1}{v^{n}} \frac{\sin v}{v}\left(\frac{\sin \Delta v}{\Delta v}\right)^{K+1} d v \\
& =\left(\frac{T}{x}\right)^{n-1} \int_{T / x}^{1 / \Delta} \frac{\sin v}{v^{n+1}}\left(1+O\left(K \Delta^{2} v^{2}\right)\right) d v+O\left(\left(\frac{T}{x}\right)^{n-1} \int_{1 / \Delta}^{\infty} \frac{1}{v^{n+1}} d v\right) \\
& =\left(\frac{T}{x}\right)^{n-1} \int_{T / x}^{1 / \Delta} \frac{\sin v}{v^{n+1}} d v+O\left(\left(\frac{T}{x}\right)^{n-1} K \Delta^{2} \int_{T / x}^{1 / \Delta} \frac{1}{v^{n-1}} d v\right)+O\left(\left(\frac{T}{x}\right)^{n-1} \Delta^{n}\right)
\end{aligned}
$$




$$
\begin{aligned}
& =\left(\frac{T}{x}\right)^{n-1} \int_{T / x}^{\infty} \frac{\sin v}{v^{n+1}} d v+O\left(K \Delta \log \frac{1}{\Delta}\right) \\
& =\frac{x}{T} \int_{1}^{\infty} \frac{\sin \frac{T y}{x}}{y^{n+1}} d y+O\left(K \Delta \log \frac{1}{\Delta}\right)
\end{aligned}
$$

because $T \Delta \leq x$. Note that the error term comes from the case $n=2$. If $n \neq 2$, we can replace the error term by $O(K \Delta)$.

LEMMA 3.4. If $F(y) \ll y^{-3 / 2+\varepsilon}$ for $y \geq 1$, then

$$
\int_{1}^{\infty} F(y) \operatorname{Re} \widehat{\Psi}_{U}\left(\frac{T y}{2 \pi x}\right) d y=\int_{1}^{\infty} F(y) \frac{\sin \frac{T}{x} y}{\frac{T}{x} y} d y+O(K \Delta) .
$$

Proof. By a change of variable $v=\frac{T y}{x}$ and (10), the left hand side is

$$
\begin{aligned}
& \frac{x}{T} \int_{T / x}^{\infty} F\left(\frac{x}{T} v\right) \frac{\sin v}{v}\left(\frac{\sin \Delta v}{\Delta v}\right)^{K+1} d v \\
& =\frac{x}{T} \int_{T / x}^{1 / \Delta} F\left(\frac{x}{T} v\right) \frac{\sin v}{v}\left(1+O\left(K \Delta^{2} v^{2}\right)\right) d v+O\left(\frac{x}{T \Delta^{K+1}} \int_{1 / \Delta}^{\infty} \frac{\left|F\left(\frac{x}{T} v\right)\right|}{v^{K+2}} d v\right) \\
& =\frac{x}{T} \int_{T / x}^{1 / \Delta} F\left(\frac{x}{T} v\right) \frac{\sin v}{v} d v+O\left(K\left(\frac{T}{x}\right)^{1 / 2-\varepsilon} \Delta^{3 / 2-\varepsilon}\right) \\
& =\frac{x}{T} \int_{T / x}^{\infty} F\left(\frac{x}{T} v\right) \frac{\sin v}{v} d v+O\left(\frac{x}{T} \int_{1 / \Delta}^{\infty} \frac{\left|F\left(\frac{x}{T} v\right)\right|}{v} d v\right)+O(K \Delta) \\
& =\int_{1}^{\infty} F(y) \frac{\sin \frac{T}{x} y}{\frac{T}{x} y} d y+O(K \Delta) .
\end{aligned}
$$

4. Proof of the theorem. Throughout this section, we assume $\tau=$ $T^{1-\varepsilon} \leq T /(\log T)^{M} \leq x, U=(\log T)^{M}$ for $M>2, H^{*}=\tau^{-2} x^{2 /(1-\varepsilon)}$, and $\Psi_{U}(t)$ is defined as in the previous section. The implicit constants in the error terms may depend on $\varepsilon$ and $M$.

Proof of Theorem 1.1. Our method is that of Goldston and Gonek [5]. Let $s=\sigma+i t$,

$$
A(s):=\sum_{n \leq x} \frac{\Lambda(n)}{n^{s}}, \quad A^{*}(s):=\sum_{n>x} \frac{\Lambda(n)}{n^{s}} .
$$

Under the Riemann Hypothesis, it follows from Theorem 3.1 of [1] (with slight modification) that 


$$
\begin{aligned}
F(x, T)= & \frac{1}{2 \pi} \int_{0}^{T} \mid \frac{1}{x}\left(A\left(-\frac{1}{2}+i t\right)-\int_{1}^{x} u^{1 / 2-i t} d u\right) \\
& +\left.x\left(A^{*}\left(\frac{3}{2}+i t\right)-\int_{x}^{\infty} u^{-3 / 2-i t} d u\right)\right|^{2} d t+O\left((\log T)^{3}\right) .
\end{aligned}
$$

Inserting $\Psi_{U}(t / T)$ into the integral and extending the range of integration to the whole real line, we have

$$
F(x, T)=\frac{1}{2 \pi x^{2}} I_{1}(x, T)+\frac{x^{2}}{2 \pi} I_{2}(x, T)+O\left(\frac{T(\log T)^{2}}{U}\right)+O\left(\frac{x^{1+6 \varepsilon}}{T}\right)
$$

where

$$
\begin{aligned}
& I_{1}(x, T)=\int_{-\infty}^{\infty} \Psi_{U}\left(\frac{t}{T}\right)\left|A\left(-\frac{1}{2}+i t\right)-\int_{1}^{x} u^{1 / 2-i t} d u\right|^{2} d t \\
& I_{2}(x, T)=\int_{-\infty}^{\infty} \Psi_{U}\left(\frac{t}{T}\right)\left|A^{*}\left(\frac{3}{2}+i t\right)-\int_{x}^{\infty} u^{-3 / 2-i t} d u\right|^{2} d t
\end{aligned}
$$

by Lemma 1 of [6] with modification $V=-T / U$ and $T-T / U$, and $W=$ $2 T / U$. The Riemann Hypothesis is assumed here so that the contribution from the cross term is estimated via Theorem 3 of [5].

We now assume the Twin Prime Conjecture defined in the previous section. By Corollary 1 of [5] (see also the calculations at the end of [5] and [6]) and Lemma 2.3, one has

$$
\begin{aligned}
I_{1}(x, T)= & \widehat{\Psi}_{U}(0) T \sum_{n \leq x} \Lambda(n)^{2} n \\
& +4 \pi\left(\frac{T}{2 \pi}\right)^{3} \int_{T / 2 \pi x}^{\infty}\left(\sum_{h \leq 2 \pi x v / T} \mathfrak{S}(h) h^{2}\right) \operatorname{Re} \widehat{\Psi}_{U}(v) \frac{d v}{v^{3}} \\
& -4 \pi\left(\frac{T}{2 \pi}\right)^{3} \int_{T / 2 \pi \tau x}^{\infty}\left(\int_{0}^{2 \pi x v / T} u^{2} d u\right) \operatorname{Re} \widehat{\Psi}_{U}(v) \frac{d v}{v^{3}} \\
& +O\left(\frac{x^{3+6 \varepsilon}}{T}\right)+O\left(x^{5 / 2+7 \varepsilon}\right) \\
= & \frac{1}{2} x^{2} \log x-\frac{1}{4} T x^{2}+4 \pi\left(\frac{T}{2 \pi}\right)^{3} \int_{T / 2 \pi x}^{\infty} \sum_{h \leq 2 \pi x v / T} \mathfrak{S}(h) h^{2} \\
& \left.-\int_{0} u^{2} d u\right) \operatorname{Re} \widehat{\Psi}_{U}(v) \frac{d v}{v^{3}}-\frac{4 \pi}{3} x^{3} \int_{T / 2 \pi \tau x}^{T e} \widehat{\Psi}_{U}(v) d v
\end{aligned}
$$




$$
\begin{aligned}
& +O\left(\frac{x^{3+6 \varepsilon}}{T}\right)+O\left(x^{5 / 2+7 \varepsilon}\right) \\
= & \frac{1}{2} T x^{2} \log x-\frac{1}{4} T x^{2}+4 \pi\left(\frac{T}{2 \pi}\right)^{3} \int_{T / 2 \pi x}^{\infty}\left(\sum_{h \leq 2 \pi x v / T} \mathfrak{S}(h) h^{2}\right. \\
& \left.-\int_{0}^{2 \pi x v / T} u^{2} d u\right) \operatorname{Re} \widehat{\Psi}_{U}(v) \frac{d v}{v^{3}}-\frac{2}{3} x^{3} \int_{0}^{T / x} \frac{\sin v}{v} d v \\
& +O\left(\frac{K T x^{2}}{(\log T)^{M}}\right)+O\left(\frac{x^{3+6 \varepsilon}}{T}\right)+O\left(x^{5 / 2+7 \varepsilon}\right)
\end{aligned}
$$

because, from (10),

$$
\begin{aligned}
\int_{T / 2 \pi \tau x}^{T / 2 \pi x} \operatorname{Re} \widehat{\Psi}_{U}(v) d v & =\int_{0}^{T / 2 \pi x} \frac{\sin 2 \pi v}{2 \pi v}\left(\frac{\sin 2 \pi \Delta v}{2 \pi \Delta v}\right)^{K+1} d v+O\left(\frac{T}{\tau x}\right) \\
& =\frac{1}{2 \pi} \int_{0}^{T / x} \frac{\sin u}{u}\left(1+O\left(K \Delta^{2} u^{2}\right)\right) d u+O\left(\frac{T}{\tau x}\right) \\
& =\frac{1}{2 \pi} \int_{0}^{T / x} \frac{\sin u}{u} d u+O\left(\frac{K \Delta^{2} T^{2}}{x^{2}}\right)+O\left(\frac{T}{\tau x}\right) .
\end{aligned}
$$

Similarly, by Corollary 2 of [5] and Lemma 2.3,

$$
\begin{aligned}
I_{2}(x, T)= & \widehat{\Psi}_{U}(0) T \sum_{x<n} \frac{\Lambda(n)^{2}}{n^{3}}+\frac{8 \pi^{2}}{T} \int_{0}^{T / 2 \pi x}\left(\sum_{1 \leq h \leq H^{*}} \frac{\mathfrak{S}(h)}{h^{2}}\right) \operatorname{Re} \widehat{\Psi}_{U}(v) v d v \\
& +\frac{8 \pi^{2}}{T} \int_{T / 2 \pi x}^{T H^{*} / 2 \pi x}\left(\sum_{2 \pi x v / T<h \leq H^{*}} \frac{\mathfrak{S}(h)}{h^{2}}\right) \operatorname{Re} \widehat{\Psi}_{U}(v) v d v \\
& -\frac{8 \pi^{2}}{T} \int_{0}^{T H^{*} / 2 \pi x}\left(\int_{2 \pi x v / T}^{H^{*}} u^{-2} d u\right) \operatorname{Re} \widehat{\Psi}_{U}(v) v d v \\
& +O\left(T^{-1} x^{-1+6 \varepsilon}\right)+O\left(x^{-3 / 2+6 \varepsilon}\right)+O\left(T^{1-\varepsilon / 2} x^{-2}\right) \\
= & \frac{T \log x}{2 x^{2}}+\frac{1}{4} \frac{T}{x^{2}}+\frac{8 \pi^{2}}{T} \int_{0}^{T H^{*} / 2 \pi x}\left(\sum_{2 \pi x v / T<h \leq H^{*}} \int^{2}\right. \\
& \left.-\int_{2 \pi x v / T}^{H^{*}} \frac{d u}{u^{2}}\right) \operatorname{Re} \widehat{\Psi}_{U}(v) v d v+O\left(\frac{x^{-1+6 \varepsilon}}{T}\right)+O\left(x^{-3 / 2+6 \varepsilon}\right) .
\end{aligned}
$$


Therefore, by a change of variable $y=\frac{2 \pi x v}{T}$ and referring to (11),

$$
\begin{aligned}
F(x, T)= & \frac{T}{2 \pi} \log x+\frac{T}{\pi} \int_{1}^{\infty}\left(\sum_{h \leq y} \mathfrak{S}(h) h^{2}-\frac{y^{3}}{3}\right) \operatorname{Re} \widehat{\Psi}_{U}\left(\frac{T y}{2 \pi x}\right) \frac{d y}{y^{3}} \\
& +\frac{T}{\pi} \int_{1}^{H^{*}}\left(\sum_{y<h \leq H^{*}} \frac{\mathfrak{S}(h)}{h^{2}}-\int_{y}^{H^{*}} \frac{d u}{u^{2}}\right) \operatorname{Re} \widehat{\Psi}_{U}\left(\frac{T y}{2 \pi x}\right) y d y \\
& +\frac{T}{\pi} \int_{0}^{1}\left(\sum_{h \leq H^{*}} \frac{\mathfrak{S}(h)}{h^{2}}-\int_{y}^{H^{*}} \frac{d u}{u^{2}}\right) \operatorname{Re} \widehat{\Psi}_{U}\left(\frac{T y}{2 \pi x}\right) y d y \\
& -\frac{x}{3 \pi} \int_{0}^{T / x} \frac{\sin v}{v} d v+O\left(\frac{K T}{(\log T)^{M-2}}\right)+O\left(\frac{x^{1+6 \varepsilon}}{T}\right) \\
& +O\left(x^{1 / 2+7 \varepsilon}\right) \\
= & \frac{T}{2 \pi} \log x+\frac{T}{\pi} I_{1}+\frac{T}{\pi} I_{2}+\frac{T}{\pi} I_{3}-\frac{x}{3 \pi} \int_{0}^{T / x} \frac{\sin v}{v} d v \\
& +O\left(\frac{K T}{(\log T)^{M-2}}\right)+O\left(\frac{x^{1+6 \varepsilon}}{T}\right)+O\left(x^{1 / 2+7 \varepsilon}\right),
\end{aligned}
$$

where $I_{1}, I_{2}$ and $I_{3}$ are the first three integrals respectively. Now,

$$
\begin{aligned}
I_{3}= & \int_{0}^{1}\left(\sum_{h \leq H^{*}} \frac{\mathfrak{S}(h)}{h^{2}}-\int_{y}^{H^{*}} \frac{d u}{u^{2}}\right) \operatorname{Re} \widehat{\Psi}_{U}\left(\frac{T y}{2 \pi x}\right) y d y \\
= & \frac{4 \pi^{2} x^{2}}{T^{2}}\left(\sum_{h \leq H^{*}} \frac{\mathfrak{S}(h)}{h^{2}}+O\left(\frac{1}{H^{*}}\right)\right) \int_{0}^{T / 2 \pi x} \operatorname{Re} \widehat{\Psi}_{U}(v) v d v \\
& -\int_{0}^{1} \operatorname{Re} \widehat{\Psi}_{U}\left(\frac{T y}{2 \pi x}\right) d y \\
= & \frac{x^{2}}{T^{2}}\left(\sum_{h=1}^{\infty} \frac{\mathfrak{S}(h)}{h^{2}}+O\left(\frac{1}{H^{*}}\right)\right) \int_{0}^{T / x} \sin u\left(1+O\left(K \Delta^{2} u^{2}\right)\right) d u \\
& -\frac{x}{T} \int_{0}^{T / x} \frac{\sin u}{u}\left(1+O\left(K \Delta^{2} u^{2}\right)\right) d u \\
= & \frac{x^{2}}{T^{2}}\left(\sum_{h \leq H^{*}} \frac{\mathfrak{S}(h)}{h^{2}}\right)\left(1-\cos \frac{T}{x}\right)-\frac{x}{T} \int_{0}^{T / x} \frac{\sin u}{u} d u+O(K \Delta)
\end{aligned}
$$

by (10), Lemma 2.4 and $T \Delta \leq x$. With the notation of $S_{\alpha}(y)$ and $T_{\alpha}(y)$, 


$$
\begin{aligned}
\text { (14) } I_{1}= & \int_{1}^{\infty} S_{2}(y) \operatorname{Re} \widehat{\Psi}_{U}\left(\frac{T y}{2 \pi x}\right) \frac{d y}{y^{3}} \\
= & \int_{1}^{\infty}\left[\frac{-1}{4 y}+\frac{\varepsilon(y)}{y}-\frac{B}{2 y}\right] \operatorname{Re} \widehat{\Psi}_{U}\left(\frac{T y}{2 \pi x}\right) d y \\
& -2 \int_{1}^{\infty} \frac{f(y)}{y^{2}} \operatorname{Re} \widehat{\Psi}_{U}\left(\frac{T y}{2 \pi x}\right) d y+2 \int_{1}^{\infty} \frac{\int_{1}^{y} f(u) d u}{y^{3}} \operatorname{Re} \widehat{\Psi}_{U}\left(\frac{T y}{2 \pi x}\right) d y \\
& +\left(\frac{B}{2}+\frac{11}{12}\right) \int_{1}^{\infty} \frac{1}{y^{3}} \operatorname{Re} \widehat{\Psi}_{U}\left(\frac{T y}{2 \pi x}\right) d y+O(K \Delta) \\
= & \int_{1}^{\infty}\left[\frac{-1}{4 y}+\frac{\varepsilon(y)}{y}-\frac{B}{2 y}\right] \operatorname{Re} \widehat{\Psi}_{U}\left(\frac{T y}{2 \pi x}\right) d y \\
& -2 \int_{1}^{\infty} \frac{f(y)}{y^{2}} \frac{\sin \frac{T}{x} y}{\frac{T}{x} y} d y+2 \int_{1}^{\infty} \frac{\int_{1}^{y} f(u) d u}{y^{3}} \frac{\sin \frac{T}{x} y}{\frac{T}{x} y} d y \\
& +\left(\frac{B}{2}+\frac{11}{12}\right) \frac{x}{T} \int_{1}^{\infty} \frac{\sin \frac{T y}{x}}{y^{4}} d y+O(K \Delta)
\end{aligned}
$$

by (5), (8) and Lemmas 3.3 and 3.4. As for $I_{2}$, note that by (4) and (6),

$$
T_{2}(z)=\frac{1}{z}+O\left(\frac{(\log z)^{2 / 3}}{z^{2}}\right)
$$

and

$$
\begin{aligned}
\sum_{y<h \leq H^{*}} \frac{\mathfrak{S}(h)}{h^{2}}-\int_{y}^{H^{*}} \frac{d u}{u^{2}} & =T_{2}(y)-T_{2}\left(H^{*}\right)-\frac{1}{y}-\frac{1}{H^{*}} \\
& =T_{2}(y)-\frac{1}{y}+O\left(\frac{\left(\log H^{*}\right)^{2 / 3}}{\left(H^{*}\right)^{2}}\right) .
\end{aligned}
$$

Therefore,

$$
\begin{aligned}
I_{2}= & \int_{1}^{H^{*}}\left(T_{2}(y)-\frac{1}{y}+O\left(\frac{\left(\log H^{*}\right)^{2 / 3}}{\left(H^{*}\right)^{2}}\right)\right) \operatorname{Re} \widehat{\Psi}_{U}\left(\frac{T y}{2 \pi x}\right) y d y \\
= & \int_{1}^{H^{*}}\left(T_{2}(y)-\frac{1}{y}\right) \operatorname{Re} \widehat{\Psi}_{U}\left(\frac{T y}{2 \pi x}\right) y d y \\
& +O\left(\frac{\left(\log H^{*}\right)^{2 / 3} x^{2}}{\left(H^{*}\right)^{2} T^{2}} \int_{T / 2 \pi x}^{T H^{*} / 2 \pi x}\left|\widehat{\Psi}_{U}(v)\right| v d v\right)
\end{aligned}
$$




$$
=\int_{1}^{\infty}\left(T_{2}(y)-\frac{1}{y}\right) \operatorname{Re} \widehat{\Psi}_{U}\left(\frac{T y}{2 \pi x}\right) y d y+O\left(\frac{1}{T^{\varepsilon}}\right)
$$

because by (15) and the formula for $\widehat{\Psi}_{U}(y)$ we have

$$
\int_{H^{*}}^{\infty} \ll x\left(\log H^{*}\right)^{2 / 3} / T H^{*} \ll 1 / T^{\varepsilon}
$$

by the definition of $H^{*}$ (and a similar estimate for the error term). Applying (6), (9) and Lemma 3.4, we get

$$
\begin{aligned}
I_{2}= & \int_{1}^{\infty}\left[\frac{-1}{4 y}-\frac{\varepsilon(y)}{y}+2 y \int_{y}^{\infty} \frac{\varepsilon(u)}{u^{3}} d u\right] \operatorname{Re} \widehat{\Psi}_{U}\left(\frac{T y}{2 \pi x}\right) d y+O\left(\frac{1}{T^{\varepsilon}}\right) \\
= & \int_{1}^{\infty}\left[\frac{-1}{4 y}-\frac{\varepsilon(y)}{y}+\frac{B}{2 y}\right] \operatorname{Re} \widehat{\Psi}_{U}\left(\frac{T y}{2 \pi x}\right) d y-2 \int_{1}^{\infty} \frac{f(y)}{y^{2}} \operatorname{Re} \widehat{\Psi}_{U}\left(\frac{T y}{2 \pi x}\right) d y \\
& +6 \int_{1}^{\infty} y \int_{y}^{\infty} \frac{f(u)}{u^{4}} d u \operatorname{Re} \widehat{\Psi}_{U}\left(\frac{T y}{2 \pi x}\right) d y+O\left(\frac{1}{T^{\varepsilon}}\right) \\
= & \int_{1}^{\infty}\left[\frac{-1}{4 y}-\frac{\varepsilon(y)}{y}+\frac{B}{2 y}\right] \operatorname{Re} \widehat{\Psi}_{U}\left(\frac{T y}{2 \pi x}\right) d y-2 \int_{1}^{\infty} \frac{f(y)}{y^{2}} \frac{\sin \frac{T}{x} y}{\frac{T}{x} y} d y \\
& +6 \int_{1}^{\infty} y \int_{y}^{\infty} \frac{f(u)}{u^{4}} d u \frac{\sin \frac{T}{x} y}{\frac{T}{x} y} d y+O(K \Delta) .
\end{aligned}
$$

Now (14) and (16) give

$$
\begin{aligned}
I_{1}+I_{2}= & -\frac{x}{2 T} \int_{1}^{\infty} \frac{\sin \frac{T y}{x}}{y^{2}} d y+\left(\frac{B}{2}+\frac{11}{12}\right) \frac{x}{T} \int_{1}^{\infty} \frac{\sin \frac{T y}{x}}{y^{4}} d y \\
& -4 \int_{1}^{\infty} \frac{f(y)}{y^{2}} \frac{\sin \frac{T}{x} y}{\frac{T}{x} y} d y+2 \int_{1}^{\infty} \frac{\int_{1}^{y} f(u) d u}{y^{3}} \frac{\sin \frac{T}{x} y}{\frac{T}{x} y} d y \\
& +6 \int_{1}^{\infty} y \int_{y}^{\infty} \frac{f(u)}{u^{4}} d u \frac{\sin \frac{T}{x} y}{\frac{T}{x} y} d y+O(K \Delta)
\end{aligned}
$$

by Lemma 3.3 again. Putting (13) and (17) into (12), we have Theorem 1.1.

\section{Proof of the corollaries}

Proof of Corollary 1.1. This follows from Theorem 1.1 as $x \leq T$ and $f(u) \ll u^{1 / 2+\varepsilon}$ by Lemma 2.2 . Note that the error term is better than that of (2) for $x$ in the given range.

Before proving Corollary 1.2, we need the following lemmas. 
LEMMA 5.1.

$\int_{1}^{\infty} \frac{\sin a x}{x^{2 n}} d x=\frac{a^{2 n-1}}{(2 n-1) !}\left[\sum_{k=1}^{2 n-1} \frac{(2 n-k-1) !}{a^{2 n-k}} \sin \left(a+(k-1) \frac{\pi}{2}\right)+(-1)^{n} \operatorname{ci}(a)\right]$ where

$$
\operatorname{ci}(x)=-\int_{x}^{\infty} \frac{\cos t}{t} d t=C_{0}+\log x+\int_{0}^{x} \frac{\cos t-1}{t} d t
$$

and $C_{0}$ is Euler's constant.

Proof. This is formula $3.761(3)$ on p. 430 of [7] which can be proved by integrating by parts repeatedly.

LEMMA 5.2. If $F(y) \ll y^{-3 / 2+\varepsilon}$ for $y \geq 1$, then for $T \leq x$,

$$
\int_{1}^{\infty} F(y) \frac{\sin \frac{T}{x} y}{\frac{T}{x} y} d y=\int_{1}^{\infty} F(y) d y+O\left(\left(\frac{T}{x}\right)^{1 / 2-\varepsilon}\right) .
$$

Proof. Since $T \leq x$, the left hand side is

$$
\begin{aligned}
\int_{1}^{x / T} F(y)(1+O & \left.\left(\left(\frac{T}{x}\right)^{2} y^{2}\right)\right) d y+O\left(\int_{x / T}^{\infty} \frac{|F(y)|}{\frac{T}{x} y} d y\right) \\
& =\int_{1}^{x / T} F(y) d y+O\left(\left(\frac{T}{x}\right)^{1 / 2-\varepsilon}\right) \\
& =\int_{1}^{\infty} F(y) d y+O\left(\int_{x / T}^{\infty}|F(y)| d y\right)+O\left(\left(\frac{T}{x}\right)^{1 / 2-\varepsilon}\right) \\
& =\int_{1}^{\infty} F(y) d y+O\left(\left(\frac{T}{x}\right)^{1 / 2-\varepsilon}\right) .
\end{aligned}
$$

Proof of Corollary 1.2. First observe that when $x$ is in the required range, the error terms in Theorem 1.1 are $O_{\varepsilon, M}\left(T /(\log T)^{M-2}\right)$. Rewrite Theorem 1.1 as

$F(x, T)=\frac{T}{2 \pi} \log x-T_{1}+T_{2}-T_{3}+T_{4}-T_{5}+T_{6}+T_{7}+O\left(\frac{T}{(\log T)^{M-2}}\right)$.

Then, by Lemmas 5.1, 5.2 and 2.4,

$$
\begin{aligned}
& T_{1}=\frac{4 x}{3 \pi} \int_{0}^{T / x}\left(1+O\left(v^{2}\right)\right) d v=\frac{4 T}{3 \pi}+O\left(\frac{T^{3}}{x^{2}}\right) \\
& T_{2}=\frac{x^{2}}{\pi T}\left(\frac{7}{4}+\frac{B}{2}+6 \int_{1}^{\infty} \frac{f(u)}{u^{4}} d u+O\left(\frac{1}{H^{*}}\right)\right)\left[\frac{1}{2}\left(\frac{T}{x}\right)^{2}+O\left(\left(\frac{T}{x}\right)^{4}\right)\right]
\end{aligned}
$$




$$
\begin{aligned}
& =\frac{T}{2 \pi}\left(\frac{7}{4}+\frac{B}{2}+6 \int_{1}^{\infty} \frac{f(u)}{u^{4}} d u\right)+O\left(T^{1-2 \varepsilon}\right)+O\left(T\left(\frac{T}{x}\right)^{2}\right), \\
& T_{3}=\frac{T}{2 \pi}\left[\frac{1}{T / x} \sin \frac{T}{x}-\operatorname{ci}\left(\frac{T}{x}\right)\right] \\
& =-\frac{T}{2 \pi} \log \frac{T}{x}-\frac{C_{0} T}{2 \pi}+\frac{T}{2 \pi}+O\left(T\left(\frac{T}{x}\right)\right), \\
& T_{4}=\left(\frac{B}{2}+\frac{11}{12}\right) \frac{x}{6 \pi}\left(\frac{T}{x}\right)^{3}\left[2\left(\frac{x}{T}\right)^{3} \sin \frac{T}{x}+\left(\frac{x}{T}\right)^{2} \sin \left(\frac{T}{x}+\frac{\pi}{2}\right)\right. \\
& \left.+\left(\frac{x}{T}\right) \sin \left(\frac{T}{x}+\pi\right)+\operatorname{ci}\left(\frac{T}{x}\right)\right] \\
& =\left(\frac{B}{2}+\frac{11}{12}\right) \frac{T}{2 \pi}+O\left(T\left(\frac{T}{x}\right)\right) \text {, } \\
& T_{5}=\frac{4 T}{\pi} \int_{1}^{\infty} \frac{f(y)}{y^{2}} d y+O\left(T\left(\frac{T}{x}\right)^{1 / 2-\varepsilon}\right), \\
& T_{6}=\frac{2 T}{\pi} \int_{1}^{\infty} \frac{\int_{1}^{y} f(u) d u}{y^{3}} d y+O\left(T\left(\frac{T}{x}\right)^{1 / 2-\varepsilon}\right) \\
& =\frac{T}{\pi} \int_{1}^{\infty} \frac{f(y)}{y^{2}} d y+O\left(T\left(\frac{T}{x}\right)^{1 / 2-\varepsilon}\right), \\
& T_{7}=\frac{6 T}{\pi} \int_{1}^{\infty} y \int_{y}^{\infty} \frac{f(u)}{u^{4}} d u d y+O\left(T\left(\frac{T}{x}\right)^{1 / 2-\varepsilon}\right) \\
& =-\frac{3 T}{\pi} \int_{1}^{\infty} \frac{f(u)}{u^{4}} d u+\frac{3 T}{\pi} \int_{1}^{\infty} \frac{f(y)}{y^{2}} d y+O\left(T\left(\frac{T}{x}\right)^{1 / 2-\varepsilon}\right) .
\end{aligned}
$$

Combining these, we get

$$
\begin{aligned}
F(x, T)= & \frac{T}{2 \pi} \log T+\frac{T}{2 \pi}\left[-\frac{8}{3}+\frac{7}{4}+\frac{B}{2}+C_{0}-1+\frac{B}{2}+\frac{11}{12}\right] \\
& +O\left(T\left(\frac{T}{x}\right)^{1 / 2-\varepsilon}\right)+O\left(\frac{T}{(\log T)^{M-2}}\right) \\
= & \frac{T}{2 \pi} \log T+\frac{T}{2 \pi}[-1-\log 2 \pi]+O\left(T\left(\frac{T}{x}\right)^{1 / 2-\varepsilon}\right) \\
& +O\left(\frac{T}{(\log T)^{M-2}}\right)
\end{aligned}
$$

which gives the corollary. 
6. Conclusion. Based on (2), (3) and Corollaries 1.1 and 1.2, we propose the following more precise Strong Pair Correlation Conjecture: For any small $\varepsilon>0$ and any large $A>1$,

$$
F(x, T)=\left\{\begin{array}{cc}
\frac{T}{2 \pi} \log x+\frac{1}{x^{2}}\left[\frac{T}{2 \pi}\left(\log \frac{T}{2 \pi}\right)^{2}-2 \frac{T}{2 \pi} \log \frac{T}{2 \pi}\right] \\
+O(x)+O\left(\frac{T}{x^{1 / 2-\varepsilon}}\right) & \text { if } 1 \leq x \leq T, \\
\frac{T}{2 \pi} \log \frac{T}{2 \pi}-\frac{T}{2 \pi}+O\left(T\left(\frac{T}{x}\right)^{1 / 2-\varepsilon}\right) & \text { if } T \leq x \leq T^{1+\varepsilon}, \\
\frac{T}{2 \pi} \log \frac{T}{2 \pi}-\frac{T}{2 \pi}+O\left(T^{1-\varepsilon_{1}}\right) & \text { if } T^{1+\varepsilon} \leq x \leq T^{A},
\end{array}\right.
$$

where $\varepsilon_{1}>0$ may depend on $\varepsilon$, and the implicit constants may depend on $\varepsilon$ and $A$.

\section{References}

[1] T. H. Chan, On a conjecture of Liu and Ye, Arch. Math. (Basel) 80 (2003), 600-610.

[2] - More precise pair correlation of zeros and primes in short intervals, J. London Math. Soc. (2) 68 (2003), 579-598.

[3] J. B. Friedlander and D. A. Goldston, Some singular series averages and the distribution of Goldbach numbers in short intervals, Illinois J. Math. 39 (1995), 158-180.

[4] D. A. Goldston, Large differences between consecutive prime numbers, thesis, Univ. of California, Berkeley, 1981.

[5] D. A. Goldston and S. M. Gonek, Mean value theorems for long Dirichlet polynomials and tails of Dirichlet series, Acta Arith. 84 (1998), 155-192.

[6] D. A. Goldston, S. M. Gonek, A. E. Özlük and C. Snyder, On the pair correlation of zeros of the Riemann zeta-function, Proc. London Math. Soc. (3) 80 (2000), 31-49.

[7] I. S. Gradshteyn and I. M. Ryzhik, Table of Integrals, Series, and Products, 6th ed., Academic Press, San Diego, CA, 2000.

[8] H. L. Montgomery, The pair correlation of zeros of the zeta function, in: Analytic Number Theory (St. Louis, MO, 1972), Proc. Sympos. Pure Math. 24, Amer. Math. Soc., Providence, RI, 1973, 181-193.

[9] H. L. Montgomery and K. Soundararajan, Beyond pair correlation, in: Paul Erdős and His Mathematics, I (Budapest, 1999), Bolyai Soc. Math. Stud. 11, János Bolyai Math. Soc., Budapest, 2002, 507-514.

Mathematics Department, Yost Hall 220

Case Western Reserve University

10900 Euclid Avenue

Cleveland, OH 44106-7058, U.S.A.

E-mail: tsz.chan@case.edu 